\title{
Image Denoising Based Improved Curvelet Threshold
}

\author{
Ahmed A. Hashim ${ }^{\dagger}$ \\ department of media technology engineering, university of information technology and communications, Iraq-baghdad, \\ dr.ahmed.hashim@uoitc.edu.iq
}

\begin{abstract}
Although wavelets are powerful as a tool in image processing it has three serious drawbacks: shift sensitivity, poor directionality and lack of phase information. Wavelets suitable for dealing with objects with point singularities; it can only capture limited directional information due to its poor orientation selectivity. Through decomposing the image into a series of high-pass and low-pass filter bands, the wavelet transform extracts directional information that capture vertical, horizontal, and diagonal activity. However, in noisy images, these three linear directions are limiting and might not capture enough directional information, like medical CT scans, which do not have strong vertical, horizontal, or diagonal directional elements.

Ridgelet transform improves Multiresolution Analysis MRA segmentation; however, they capture structural information of an image based on multiple radial directions in the frequency domain. Line singularities in ridgelet transform provides better edge detection than its wavelet counterpart. One limitation to use ridgelet in image segmentation is that ridgelet is most effective in detecting linear radial structures, which are not dominant in images. The curvelet transform is a recent extension of ridgelet transform that overcome ridgelet weaknesses. Curvelet is proven to be particularly effective at detecting image activity along curves instead of radial directions which are the most comprising objects of images. However, the fact that at sufficiently fine scales, a curved edge is almost straight, and so to capture curved edges, one ought to be able to deploy ridgelets in a localized manner, at sufficiently fine scales.

In this paper, a new method is used combining the Window Shrink threshold and Bayes Shrink threshold based on Curvelet transform to enhance removing Additive Weight Gaussian Noise AWGN noise from image. It has better PSNR than the traditional Curvelet that uses each threshold alone, and the images gotten by this method is better and outperform that of the traditional curvelet and wavelet methods and the results reported here are promising.
\end{abstract}

Keywords: Image denoising, curvelet transform, wavelet transform, ridgelet transform, Peak signal to noise ration PSNR, Mean square error MSE.

\section{Introduction}

An image is often corrupted by noise in its acquisition or transmission. The goal of denoising is to remove the noise while retaining as much as possible the important image features [1]. Traditionally, this is achieved by linear processing such as Wiener filtering or Edge preserving smoothing algorithm, however, new methods uses nonlinear techniques like wavelet, ridgelet, and curvelet perform much better than the liner one [2, 3, 4]. In [2] they proved mathematically that with optimal choice of threshold in wavelet transform, near-optimal properties can be reconstructed from the signal and with better rate of convergence than linear techniques.

The success of wavelets is mainly due to the good performance for piecewise smooth functions in one dimension. Unfortunately, such is not the case in two dimensions. In essence, wavelets are good at catching point singularities, but two-dimensional piecewise smooth signals resembling images have one-dimensional singularities. i.e. smooth regions are separated by edges, and while edges are discontinuous across, they are typically smooth curves. Intuitively, wavelets in two dimensions are obtained by a tensor-product of one dimensional wavelets and they are thus good at isolating the discontinuity across an edge, but will not see the smoothness along the edge [5].

To overcome the weakness of wavelets in higher dimensions, Candès and Donoho $[2,7]$ recently pioneered a new system of representations named ridgelets which deal effectively with line singularities in 2-D. "The idea is to map a line singularity into a point singularity using the Radon transform. Then, the wavelet transform can be used to effectively handle the point singularity in the Radon domain". However, since most of the images are carved in nature, a modification of the ridgelet is proposed by Candès and Donoho [2], the curvelet which deals with image activity along curves instead of radial directions which are the most comprising objects of images. Since a curved edge is almost straight when dealing with it in fine scale, A resent modification is to use curve let then subdivided it into small block, each block can be assumed linear and hence applying ridgelet on it [6]. The proposed method is based on improving the threshold of curvelet transform and hence increasing the PSNR in image denoising. 


\section{Wavelet, Ridgelet, and Curvelet Transforms}

\subsection{Wavelet Transform}

During the last decade, a wide range of applications, including numerical analysis, image/video processing, and telecommunication has been recognized wavelet transform as a powerful tool [6]. "The advantage of wavelet is that wavelet performs an MRA of a signal with localization in both time and frequency" $[1,11]$. In addition to this, fewer wavelet basis vectors is required in the wavelet domain than sine cosine basis vectors to achieve a comparable approximation when functions with discontinuities and functions with sharp spikes is available. Discrete wavelet transform (DWT) can be implemented as a set of highpass and low-pass filter banks. In standard wavelet decomposition, the output from the low-pass filter can be then decomposed further, with the process continuing recursively in this manner. According to [5], DWT can be mathematically expressed by:

$a^{j}(n)=\sum_{i=0}^{L-1} l(i) \cdot a^{j-1}(2 n-i), \quad 0 \leq n<N_{j}$

$d^{j}(n)=\sum_{i=0}^{L-1} h(i) \cdot d^{j-1}(2 n-i), 0 \leq n<N_{j}$

While $l(i)$ and $h(i)$ represent the coefficients of low-pass and high-pass filters, respectively, the coefficients $a^{j}(n)$ and $d^{j}(n)$ refer to approximation and detailed components in the signal at decomposition level $\mathrm{j}$, respectively.

DWT decomposes the signal into a set of resolution related views. At each scale $j$, the wavelet decomposition of an image creates a set of coefficient values $w_{j}$ with an overall mean of zero. $w_{j}$ contains the same number of voxels as the original image.

For images, 1D-DWT can be readily extended into $2 \mathrm{D}$. Either in standard 2D wavelet decomposition; where the image rows are fully decomposed, with the output being fully decomposed column wise or in nonstandard wavelet decomposition; where all the rows are decomposed by one decomposition level followed by one decomposition level of the columns, figure 1 illustrates the filter structure of 2D-DWT.

Depending on filter coefficients and the number of those coefficients, wavelet uses a set of filters to decompose images. Haar wavelet filter (HWF) is the most popular wavelet filter which takes the averages and differences from the low- and high-pass filters, respectively [8]. Figure 2 illustrates an example of applying 2D-DWT using HWF on an image for 2 levels of decompositions.

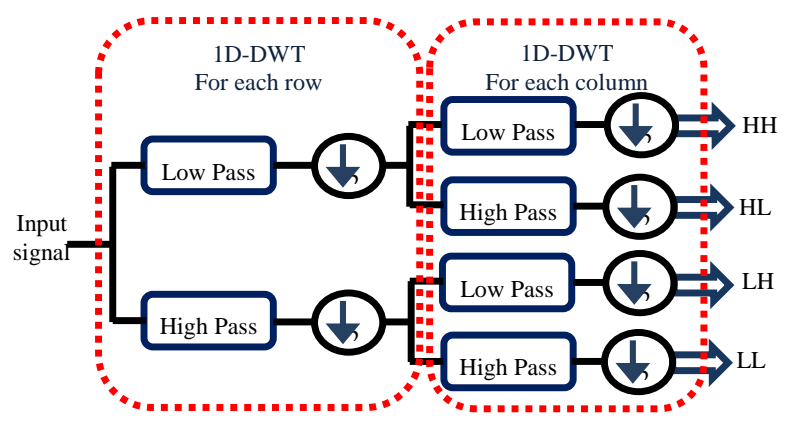

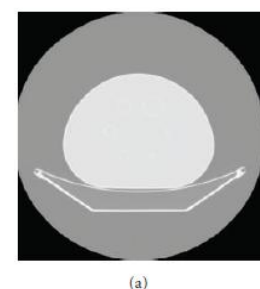
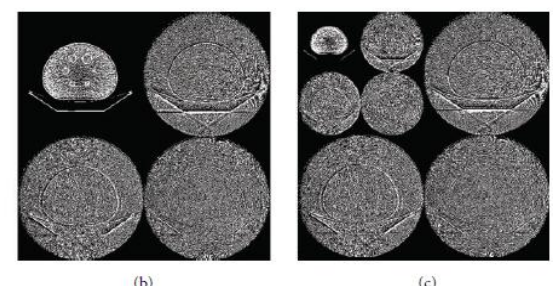

Fig. 2: 2D-DWT, Original image (a), first decomposition level (b), second decomposition level

\subsection{Ridgelet Transform}

In 1998, Donoho introduced the ridgelet transform [2] "continuous ridgelet transform (CRT) can be defined from a 1D wavelet function oriented at constant lines and radial directions". Ridgelet transform [3, 11, 17] has superior performance over wavelets. While wavelets very successful in applications like denoising of images containing zero dimensional or point singularities; it do not isolate the smoothness along edges that occurs in images, and thus more appropriate for the reconstruction of sharp point singularities than lines or edges. These shortcomings of wavelet are well addressed by the ridgelet transform, generally speaking, wavelets detect objects with point singularities, while ridgelets are able to represent objects with line singularities.

"The finite ridgelet transform (FRIT) computed in two steps: a calculation of discrete radon transform and an application of a wavelet transform. The finite radon transform (FRAT) is computed in two steps: a calculation of 2D Fast Fourier Transform (FFT) for the image and an application of a 1D inverse fast Fourier transform (IFFT) on each of the radial directions of the radon projection" [3, 5].

Applying FRAT on image means setting of projections of the image taken at different angles to map the image space to projection space. For discrete images, a projection is computed by summation of all data points that lie within specified unit-width strips; those lines are defined in a finite geometry [5]. Depending on [5], FRAT of a real function on the finite grid $\mathrm{Z}_{\mathrm{P}}^{2}$ is defined in

$\mathrm{r}_{\mathrm{k}}[\mathrm{l}]=\operatorname{FRAT}_{\mathrm{f}}(\mathrm{k}, \mathrm{l})=\frac{1}{\sqrt{\mathrm{P}}} \sum_{(\mathrm{i}, \mathrm{j}) \in \mathrm{L}(\mathrm{k}, \mathrm{l})} \mathrm{f}(\mathrm{i}, \mathrm{j})$

(2)

Here, $\mathrm{L}(\mathrm{k}, \mathrm{l})$ denotes the set op points that make up a line on the lattice $\mathrm{Z}_{\mathrm{P}}^{2}$ as in

$\mathrm{L}(\mathrm{k}, \mathrm{l})=\left\{(\mathrm{i}, \mathrm{j}): \mathrm{j}=\mathrm{k}_{\mathrm{i}}+\mathrm{l}(\bmod \mathrm{P}), \mathrm{i} \in \mathrm{Z}_{\mathrm{P}}\right\}, \quad 0 \leq \mathrm{k}<\mathrm{P}$, $L(P, l)=\left\{(l, j): j \in Z_{P}\right\}$.

(3)

To compute the $\mathrm{K}^{\text {th }}$ radon projection (the $\mathrm{K}^{\text {th }}$ row in the array), all pixels of the original image need to be passed once and use P histogrammers: one for every pixel in the row. At the end, all $\mathrm{P}$ histogrammed values are divided by $\mathrm{K}$ to get the average values. Once the wavelet and radon transforms have been implemented, the ridgelet transform is straightforward. Each output of the radon projection is simply passed through the wavelet transform before it 
reaches the output multiplier. As shown in Figure 3, since FRAT maps a line singularity into point singularity, FRAT used as a basic building block of ridgelet, and the wavelet transform has used to effectively detect and segment the point singularity in radon domain [7].

Continuous ridgelet transform is similar to the continuous wavelet transform except that point parameters (x, y) in the Cartesian grid (Figure 4(a)) which perform pixels in the image or an entry in a $2 \mathrm{D}$ matrix are now replaced by line parameters $(\beta, \theta)$, where $\beta$ is the intercept and $\theta$ is the angle. Figure 4(b) illustrates the radial grid in ridgelet transform; however, straight lines evaluate the image in the frequency domain.

Using ridgelet transformation on images was not promising, since most images comprised from curves which are still not singularity points after applying radon transform, Ridgelet transform can be used in other applications, where images contain edges and straight lines. Curvelet transform has been introduced to solve this problem; it deals with higher singularities compared to wavelet and ridgelet transforms.

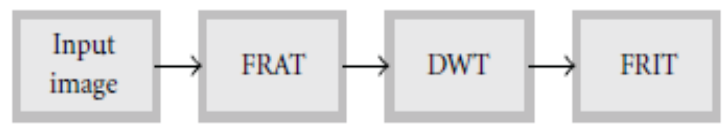

Fig.3: RFIT block diagram

\begin{tabular}{|c|c|c|c|c|c|c|}
\hline$x$ & $x$ & $x$ & $x$ & $x$ & $x$ & $x$ \\
\hline$x$ & $x$ & $x$ & $x$ & $x$ & $x$ & $x$ \\
\hline$x$ & $x$ & $x$ & $x$ & $x$ & $x$ & $x$ \\
\hline$x$ & $x$ & $x$ & $x$ & $x$ & $x$ & $x$ \\
\hline$x$ & $x$ & $x$ & $x$ & $x$ & $x$ & $x$ \\
\hline$x$ & $x$ & $x$ & $x$ & $x$ & $x$ & $x$ \\
\hline$x$ & $x$ & $x$ & $x$ & $x$ & $x$ & $x$ \\
\hline
\end{tabular}

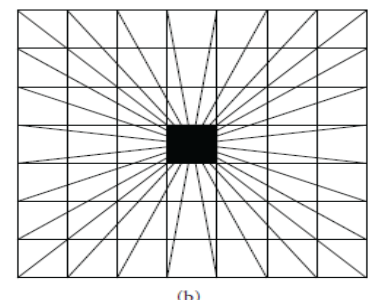

Fig.4: Wavelet and Ridgelet Parameters

\subsection{Curvelet Transform}

The curvelet transform was first introduced in 2000 by Cand'es and Donoho [2,7], which used a complex series of steps involving the ridgelet analysis of the radon transform of an image with very slow performance; a newer method was developed that easier to use and understand. The use of ridgelet transform as a preprocessing step of curvelet was discarded, thus reducing the amount of redundancy in the transform and increasing the speed considerably. This improved version of curvelet transform was known as Fast Discrete Curvelet Transform (FDCT). "Curvelet aims to deal with interesting phenomena occurring along curved edges in a $2 \mathrm{D}$ image. As illustrated in figure 5, curvelet needs fewer coefficients for representation, and the edge produced from curvelet is smoother than wavelet edge" $[6,18]$
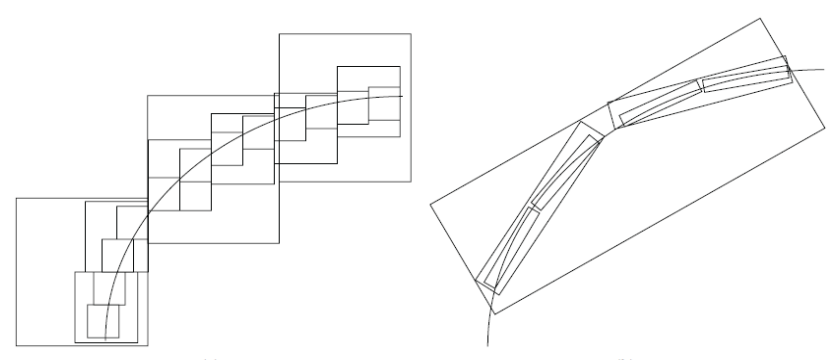

Fig.5: An approximating comparison between wavelet (a) and curvelet (b)

While the ridgelet transform steps can be summarized in figure 6 , figure 7 shows the curvelet steps, from which one can understand the main differences between the two transforms, and how the first version of curvelet transform is built based on ridgelet transform. Figure 8 from the other hand shows how the image's curves is subdivided into small parts each of them treated as if straight line with significant and insignificant coefficients as seen in figure 9

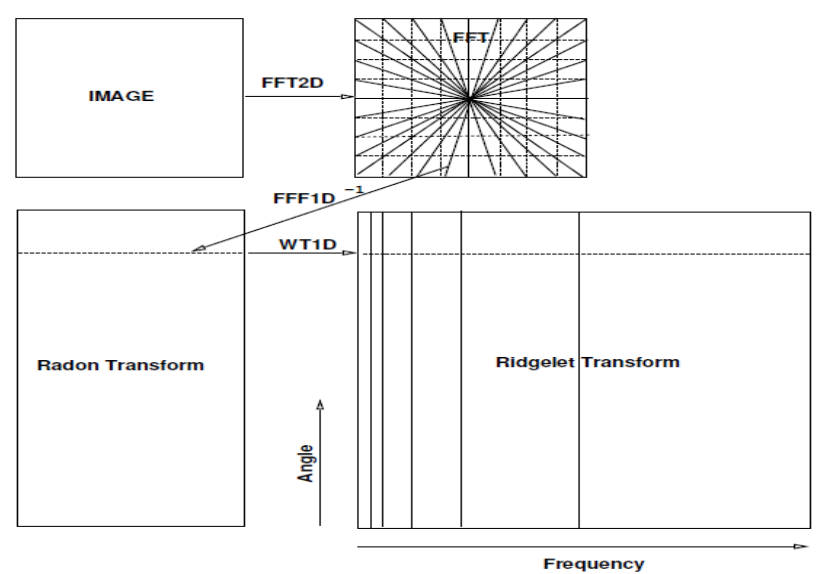

Fig.6: DRT flowchart. Each of the $2 \mathrm{n}$ radial lines in the Fourier domain is processed separately. The 1-D IFFT is calculated along each radial line followed by a 1-D nonorthogonal wavelet transform. In practice, the onedimensional wavelet coefficients are directly calculated in the Fourier space

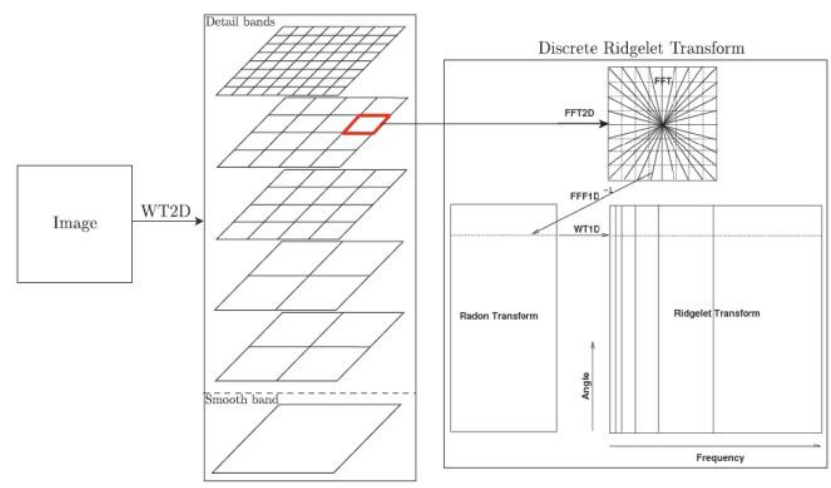

Fig.7: First Generation Discrete Curvelet Transform (DCTG1) flowchart. The figure illustrates the decomposition of the original image into sub-bands followed by the spatial partitioning of each sub-band. The ridgelet transform is then applied to each block 


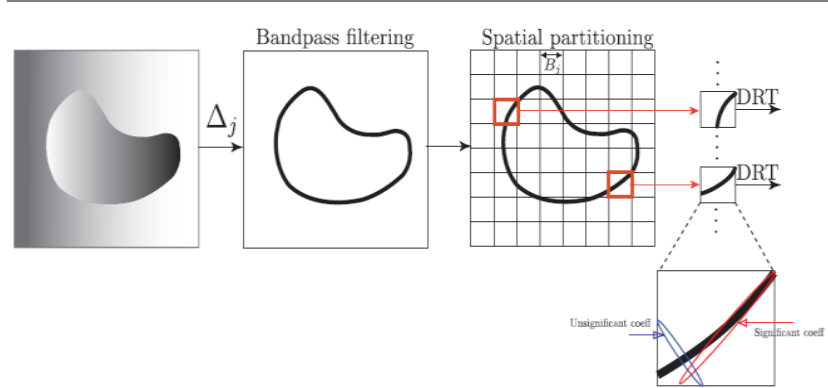

Figure 8: Local ridgelet transform on bandpass filtered image. At fine scales, curved edges are almost straight lines

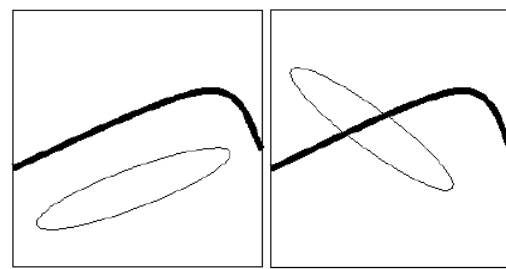

(a)

(b)

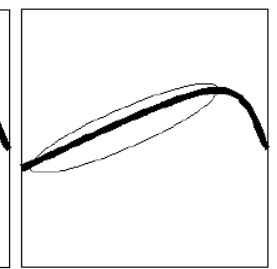

(c)
Figure 9: curvelet types. Type A The curvelet coefficient magnitude will be zero (a). Type B The curvelet coefficient magnitude will be close to zero (b). Type C The curvelet coefficient magnitude will be much larger than zero (c).

\subsubsection{Continuous - time curvelet Transform}

This transform works in two dimensions with spatial variable $x$, frequency domain variable $\omega$, and the frequency-domain polar coordinates $r$ and $\theta$. It can be defined by a pair of windows, radial window $\{W(r)\}$, and angular window $\{V(t)\}$. As illustrated in [7], these windows will always obey the admissibility conditions.

$$
\begin{aligned}
& \sum_{j=-\infty}^{\infty} W^{2}\left(2^{j} r\right)=1, \quad r \in\left(\frac{3}{4}, \frac{3}{2}\right), \\
& \sum_{j=-\infty}^{\infty} V^{2}(t-l)=1 \quad t \in\left(-\frac{1}{2}, \frac{1}{2}\right)
\end{aligned}
$$

A polar "wedge" represented by $U_{j}$ is supported by the radial window $\{W(r)\}$ and angular window $\{V(r)\}$. Equation (5) defines $U_{j}$ in the Fourier domain

$$
\begin{aligned}
& U_{j}(r, \theta) \\
& =2^{-\frac{3 j}{4}} W\left(2^{-j} r\right) V\left(\frac{2^{[j / 2]} \theta}{2 \pi}\right)
\end{aligned}
$$

Equation (6) define the curvelet transform as a function of $\left\{x=\left(x_{1}, x_{2}\right)\right\}$ at scale $2^{-j}$, orientation $\theta_{l}$ and position $x_{k}(j, l)$, where $R_{\theta}$ is the rotation in radians. Figure 10 illustrates the induced tiling of the frequency plane and the spatial Cartesian grid associated with a given scale and orientation, and shaded area represents the polar wedge by $U_{j}$ $\varphi_{j, l, k}(x)=\varphi_{j}\left(R_{\theta_{l}}\left(x-x_{k}^{(j, l)}\right)\right)$

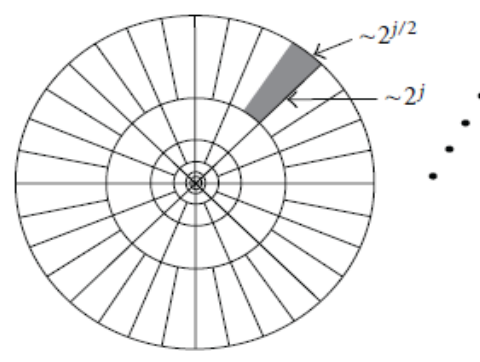

(a)

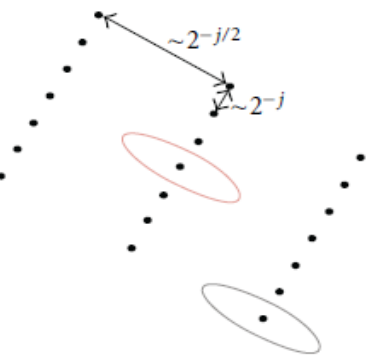

(b)
Fig.10: Curvelet tiling of space and frequency.The induced tiling of the frequency plane (a).The spatial Cartesian grid associated with a given scale and orientation (b)

\subsubsection{Fast Discrete Curvelet Transform}

Curvelet transform implementation based on Wrapping of Fourier samples takes an image as an input in the form of a Cartesian array $f[m, n]$, where $0 \leq m<M, 0 \leq n<N$ where $M$ and $N$ are the dimensions of the array. As illustrated in equation (7), the outputs will be a collection of curvelet coefficients $c^{D}\left(j, l, k_{l} k_{2}\right)$ indexed by a scale $j$, an orientation $l$ and spatial location parameters $k_{l}$ and $k_{2}$ [5].

$$
c^{D}\left(j, l, k_{1} k_{2}\right)=\sum_{0 \leq n<N}^{0 \leq m<M} f[m, n] \varphi_{j, l, k_{1} k_{2}}^{D}[m, n]
$$

Each $\varphi_{j, l, k_{1} k_{2}}^{D}$ is a digital curvelet waveform, superscript D stands for "digital." "These approach implementations are the effective parabolic scaling law on the subbands in the frequency domain to capture curved edges within an image in more effective way" [5]. Figure 11 illustrates the whole image represented in spectral domain in the form of rectangular frequency tiling by combining all frequency responses of curvelets at different scales and orientations. From figure 11 it can be seen that curvelets are needle like elements at higher scale, also the curvelet becomes finer and smaller in the spatial domain and more sensitivity to curved edges as the resolution level is increased, thus effectively capture the curves in an image, and curved singularities can be well-approximated with fewer coefficients [7].

curvelet transform is usually implemented in the (5frequency domain in order to achieve a higher level of efficiency. This means that a 2D FFT is applied to the image. For each scale and orientation, a product of $U_{j l}$ "wedge" is obtained; the result is then wrapped around the origin, and 2D IFFT is then applied resulting in discrete curvelet coefficients. The discrete curvelet transform can be described as:

Curvelet transform $=$ IFFT[FFT $\quad$ (Curvelet) $\times$ FFT(Image)] (8) 


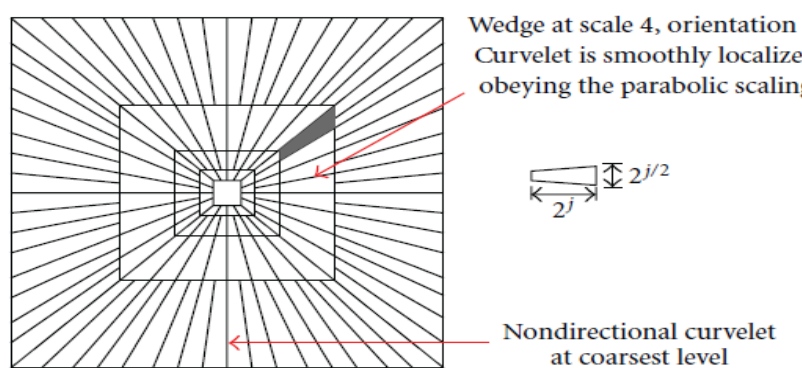

Fig.11: 5-level curvelet digital tiling of an image

The following are the steps of applying wrapping based FDCT algorithm [12].

1- Apply the 2D FFT to an image to obtain Fourier samples

$\hat{f}[m, n], \quad \frac{n}{2} \leq m, n<\frac{n}{2}$

2- For each scale $\mathrm{j}$ and angle 1 , form the product

$$
\widetilde{U}_{j, l}[m, n] \hat{f}[m, n]
$$

3- Wrap this product around the origin and obtain

$$
\tilde{f}_{j, l}[m, n]=W\left(\widetilde{U}_{j, l} \hat{f}\right)[m, n],
$$

where the range for $\mathrm{m}, \mathrm{n}$, and $\theta$ is now $0 \leq \mathrm{m}<2^{\mathrm{j}}, 0 \leq \mathrm{n}<$ $2^{\mathrm{j} / 2}$, and $-\pi / 4 \leq \theta<\pi / 4$

4- Apply IFFT to each $\tilde{f}_{j, l}$, hence collecting the discrete coefficients $c^{D}\left(j, l, k_{1} k_{2}\right)$.

Curvelet transform is a multiscale transform such as wavelet, with frame elements indexed by scale and location parameters. Wavelets are only suitable for objects with point singularities, Ridgelets are only suitable for objects with line singularities, while curvelets have directional parameters and its pyramid contains elements with a very high degree of directional specificity and obeying a special scaling law, where the length and the width of frame elements are linked using:

Width

(12)

length ${ }^{2}$

Discrete curvelet transform in the spectral domain utilizes the advantages of FFT. During FFT, both image and curvelet at a given scale and orientation are transformed into the Fourier domain. The convolution of the curvelet with the image in the spatial domain then becomes their product in the Fourier domain. At the end of this computation process, a set of curvelet coefficients are obtained by applying IFFT to the spectral product. This set contains curvelet coefficients in ascending order of the scales and orientations.

\section{Proposed Algorithm}

One of most critical issues in curvelet, wavelet, ridgelet and many other transforms is how to define the optimal threshold value that maximize the probability of reconstructing the original signal/image[1, 4, 10, 13, 15]. This section describes the proposed image denoising algorithm, which achieves near optimal soft thresholding in the curvelet domain for recovering original signal from the noisy one. The algorithm merges between two thresholding techniques used by curvelet:

\section{i. Window Shrink Method [14]}

In this method the steps of finding the threshold value can be summarized by the following steps:

1) Set $\mathbf{d}_{i, j}$ is the parameter which is from curvelet transformed noise image; choose a $\mathbf{d}_{\mathbf{i}, \mathbf{j}}$ centered window of $n \times n$ as the processing subject.

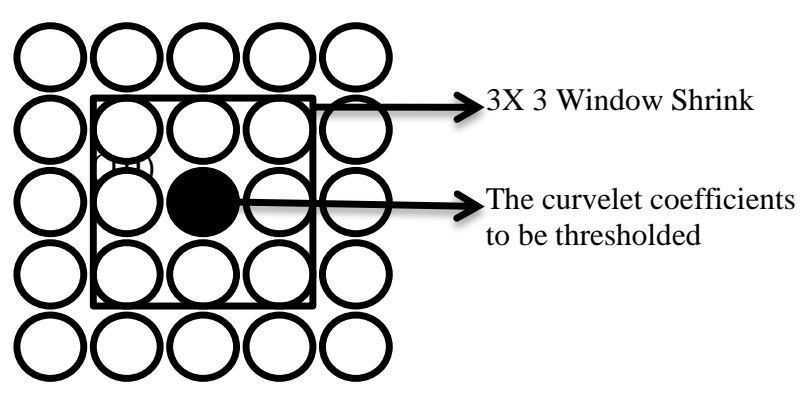

Fig.12: choosing the processing curvelet coefficient centered in the $n * n$ window

2) The sum of all the parameter's square in the $n \times n$ window is calculated

$$
S_{i, j}^{2}=\sum_{p=i-\frac{(n-1)}{2}}^{i+\frac{(n-1)}{2}} \quad \sum_{q=j-\frac{(n-1)}{2}}^{j+\frac{(n-1)}{2}} \quad d_{p, q}^{2}
$$

3) Set Symbolic function:

$$
\eta=\sqrt{2 \sigma^{2} \log n^{2}}
$$

4) $\sigma$ is the variance of Gaussian white noise in the image, then shrinking processing parameter is

$$
\begin{aligned}
& \alpha_{i, j}=I\left(1-\frac{\eta^{2}}{S_{i, j}^{2}}\right) \\
& I(X)= \begin{cases}X, & X \geq 0 \\
0, & X<0\end{cases}
\end{aligned}
$$

5) Then the thresholded parameter can be calculated as $d_{i, j}^{\prime}=d_{i, j} * \alpha_{i, j}$

(17)

\section{ii. Bayes Shrink method [16]}

In this method the steps of finding the threshold value can be summarized by the following steps: 
1) In this method $\sigma_{D}^{2}$ is the variance of an image containing noise, $\sigma^{2}$ is the variance of noise, and $\sigma_{X}^{2}$ is the original image's variance.

2) Now, noise variance is

$$
\sigma=\frac{\text { Median }\left|d_{i, j}\right|}{0.6745}
$$

$$
\sigma_{D}^{2}=\frac{1}{M N} \sum_{i, j} d_{i, j}^{2}
$$

3) The variance of original image is calculated by

$$
\sigma_{X}=\sqrt{\left|\sigma_{D}^{2}-\sigma^{2}\right|}
$$

4) Setting Threshold is $\frac{\sigma^{2}}{\sigma_{X}^{2}}$ then begin the processing of removing noise

\section{iii. Combination of Window shrink and Bayes shrink (the proposed method)}

In this proposed method the steps of finding the threshold value (improved threshold) can be summarized by the following steps:

1) Estimating the variance of the original picture $\sigma_{X}^{2}$ using Bayes shrink method (equations 18-20).

2) Calculating $\eta$ using $\sigma_{X}^{2}$ instead of $\sigma^{2}$, so that equation (14) becomes:

$\eta=\sqrt{2 \sigma_{X}^{2} \log n^{2}}$

3) Using equation (15) to find shrink parameter $\alpha_{i, j}$

4) Using equations (16-17) to filter out the noise coefficient.

\section{Results}

First of all a MATLAB computer program is written for wavelet, ridgelet and traditional curvelet to apply them on noisy image with $\sigma=20$ in order to compare between different transforms efficiency in denoising, the results are shown in figure 13. It is clear from the results that the traditional curvelet transform has a superior efficiency over the other transforms and one can see that the curves details of the image is better reconstructed using curvelet transform. Table 1 shows the values of PSNR, MSE of these transforms. After that a MATLAB program is written to compare between traditional curvelet transform TCT and improved curvelet transform ICT in terms of their efficiency in image denoising for different values of noise standard deviation, the results are shown in figure 14,15 and in table 2, where it seems very clear that the new thresholding method boost the PSNR asymptotically.
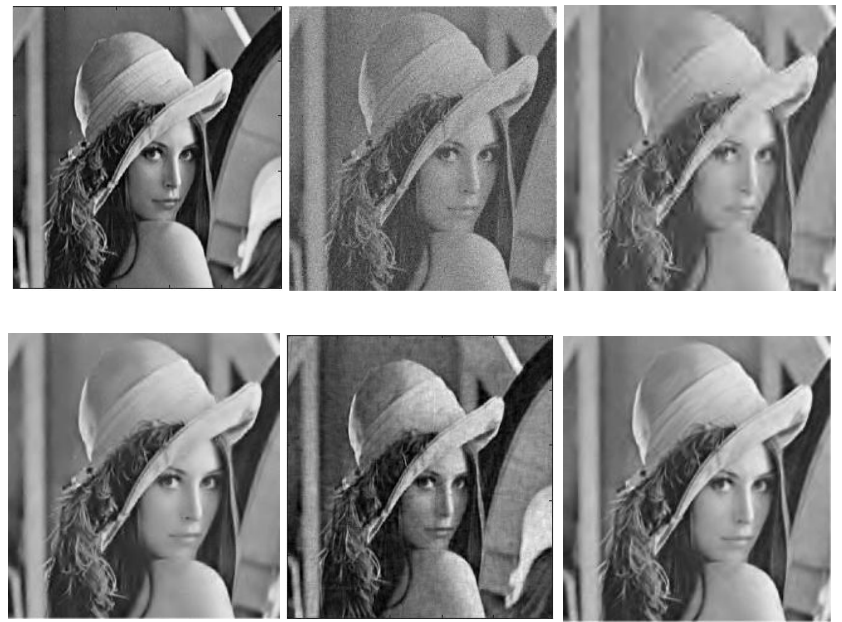

Fig.13: (a) Noisy free image (b) noisy image with $\sigma=20$. Filtered images using:(c) decimated wavelet transform (d) undecimated wavelet transform (e) ridgelet transform and (f) curvelet transform

Table 1: comparison between different transforms for different values of noise standard deviation $\sigma$

$\sigma$ of Noisy Decimated Undecimated Ridgelet Traditional image PSNR wavelet wavelet transform Curvelet

\begin{tabular}{cccccc}
\hline 5 & 34.15 & 35.3 & 37.06 & 37.19 & 37.9 \\
10 & 28.13 & 31.4 & 33 & 33.13 & 34.554 \\
20 & 22.1 & 25.6 & 29.21 & 29.32 & 31.47 \\
30 & 18.6 & 22.74 & 26.9 & 27.03 & 29.95 \\
40 & 16.09 & 22.05 & 25.62 & 25.8 & 27.645 \\
50 & 14.15 & 21.8 & 24.47 & 24.65 & 27.645 \\
60 & 12.57 & 21.23 & 23.57 & 23.74 & 26.966 \\
\hline
\end{tabular}
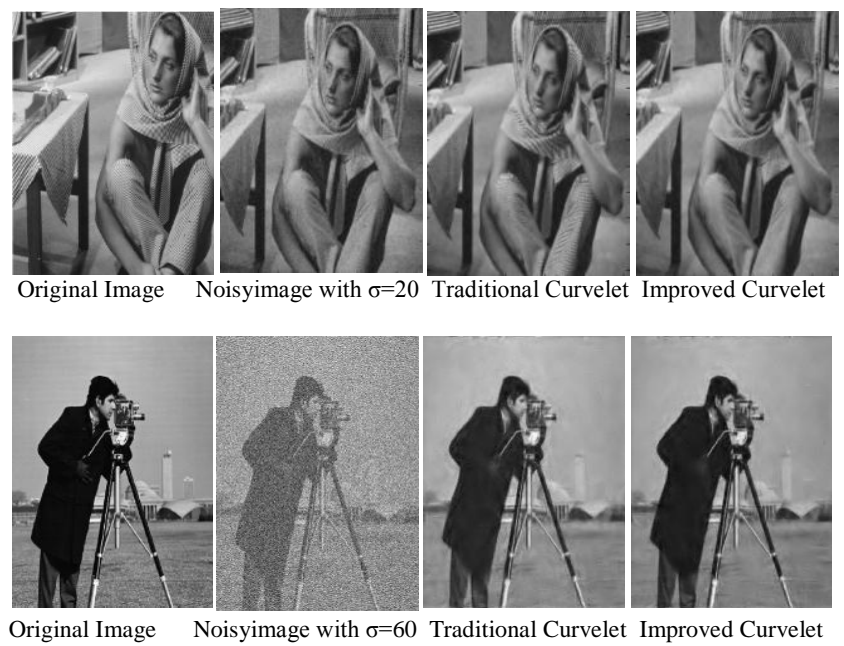

Fig.14: Comparison between traditional curvelet and improved curvelet thresholding 


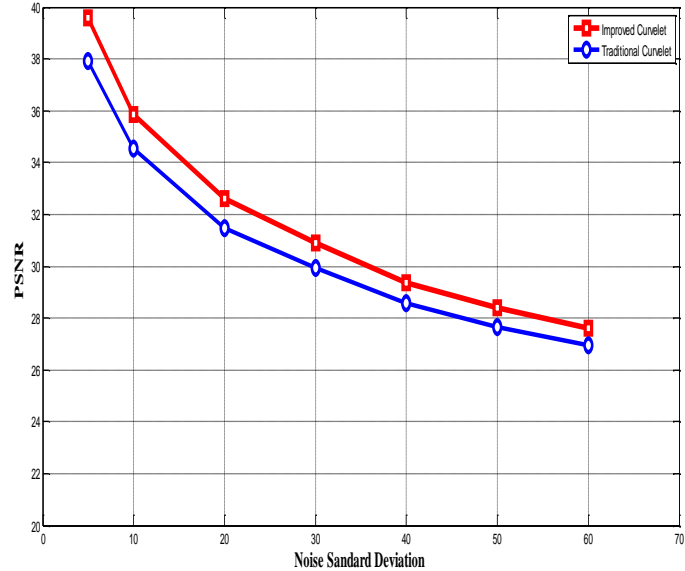

Fig.15: PSNR of Traditional curvelet versus PSNR of Improved Curvelet thresholding for different values of $\sigma$

Table 2: comparison between traditional and improved curvelet for different values of noise standard deviation

\begin{tabular}{cccc}
$\begin{array}{c}\sigma \text { of noisy } \\
\text { image }\end{array}$ & $\begin{array}{c}\text { Noisy image } \\
\text { PSNR }\end{array}$ & $\begin{array}{c}\text { Traditional } \\
\text { Curvelet }\end{array}$ & $\begin{array}{c}\text { Improved } \\
\text { curvelet }\end{array}$ \\
\hline 5 & 34.15 & 37.9 & 39.1 \\
10 & 28.13 & 34.554 & 35.65 \\
20 & 22.1 & 31.47 & 32.4 \\
30 & 18.6 & 29.95 & 30.82 \\
40 & 16.09 & 27.645 & 29.35 \\
50 & 14.15 & 27.645 & 28.42 \\
60 & 12.57 & 26.966 & 27.6 \\
\hline
\end{tabular}

\section{Conclusions}

In this paper a method was introduced to enhance the image denoising through using improved curvelet thresholding. To overcome the disadvantages of the wavelet transform along the curves in the images the curvelet transform is used and it gives higher PSNR. A new method of combination of the Window Shrink and Bayes Shrink based on Curvelet transform is used to remove AWGN noise from image. It has better PSNR. So the image we got by this method is better than that of the traditional curvelet methods and hence better than wavelet and ridgelet methods. There is no general transform that can be considered as the best one for all kinds of images, the success of wavelets is mainly due to the good performance for piecewise smooth functions in one dimension the wavelet is better in point singularity and gives no information for the direction and orientation, the ridgelet from the other hand is better than wavelet in line singularity, and since most of the images are curved in nature; the curvelet transform is better in reconstructing curved images, however it is important to mention that the time consumed using curvelet transform (traditional and improved) is larger than that of wavelet and ridgelet transform.

\section{References}

[1] S. Grace Chang, Bin Yu, and Martin Vetterli, "Adaptive Wavelet Thresholding for Image Denoising and Compression", IEEE Transactions on Image Processing, Vol. 9, No. 9, pp 1532-1546, September 2000

[2] Jean-Luc Starck, Emmanuel J. Candes, David L. Donoho, "The Curvelet Transform for Image Denoising", Stanford University, Technical report, November 15, 2000

[3] M.J. Fadili , J.-L. Starck, "Curvelets and Ridgelets", Technical report, Caen Cedex, France, October 24, 2007

[4] Aarti S.Gaikwad, Rajarshri Shahu, "Image Denoising Using Digital Image Curvelet", Journal of Information Engineering and Applications, ISSN 2224-5758 (print) ISSN 2224-896X (online), Vol 1, No.2, pp. 23-29, 2011

[5] Shadi AlZubi, Naveed Islam, and Maysam Abbod, "Multiresolution Analysis Using Wavelet, Ridgelet, and Curvelet Transforms for Medical Image Segmentation" Research Article, Hindawi Publishing Corporation, International Journal of Biomedical Imaging, Article ID 136034, pp. 1-18, 2011

[6] Emmanuel Cand es, Laurent Demanet, David Donoho and Lexing Ying, "Fast Discrete Curvelet Transforms" , Applied and Computational Mathematics, Caltech, Pasadena, Technical report, March 2006

[7] Jean-Luc Starck, Fionn Murtagh, Emmanuel J. Candès, and David L. Donoho, "Gray and Color Image Contrast Enhancement by the Curvelet Transform", IEEE Transactions on Image Processing, Vol. 12, No. 6, pp. 706 -717, JUNE 2003

[8] Lakhwinder Kaur, Savita Gupta, R.C. Chauhan, "Image Denoising using Wavelet Thresholding", SLIET, Longowal, 2003

[9] Florian Luisier , Ce'dric Vonesch , Thierry Blu , Michael Unser, " Fast interscale wavelet denoising of Poisson-corrupted images", Elsevier, Signal Processing, 2010

[10]P. Karthikeyan, et al, "Hybrid Noise Removal in Color Images using Wavelet Shrinkage Approaches of PURE-LET and Neighshrink SURE", International Journal of Emerging Trends in Electrical and Electronics (IJETEE), Vol. 2, Issue. 3, pp 57-61, April-2013

[11]Bo Zhang, Jalal M. Fadili, and Jean-Luc Starck, "Wavelets, Ridgelets, and Curvelets for Poisson Noise Removal", IEEE Transactions on Image Processing, Vol. 17, No. 7, pp 1093-1108, July 2008

[12]Jean-Luc Starck, Emmanuel J. Candès, and David L. Donoho, "The Curvelet Transform for Image Denoising", IEEE Transactions on Image Processing, Vol. 11, No. 6, pp 670-684, June 2002

[13]A. Djimli, D. Tchiotsop and R. Tchinda, "Analysis of Interest Points of Curvelet Coefficients Contributions of Microscopic Images and Improvement of Edges", Signal \& Image Processing : An International Journal (SIPIJ) Vol.4, No.2, pp. 1-9, April 2013

[14]Jianwei Ma and Gerlind Plonka, "Combined Curvelet 
Shrinkage and Nonlinear Anisotropic Diffusion", IEEE TRANSACTIONS ON IMAGE PROCESSING, VOL. 16, NO. 9, pp 2198-2206, SEPTEMBER 2007

[15]Laurent Jacques, Laurent Duval, Caroline Chaux, Gabriel Peyré, "Curvelets, contourlets, shearlets, *lets, etc.: multiscale analysis and directional wavelets for images", UCL, IFPEN, AMU, Dauphine, Seminar, October 2013

[16]Nezamoddin Nezamoddini-Kachouie, Paul Fieguth, and Edward Jernigan, " BayesShrink Ridgelets for Image Denoising", International Conference on Image Analysis and Recognition ICIAR, Springer-Verlag Berlin Heidelberg, pp 163-170, 2004

[17]Minh N. Do, and Martin Vetterli, "The Finite Ridgelet Transform for Image Representation",IEEE Transactions on Image Processing,Vol.12,No.1,pp1628,Jan2003

[18]Brian Eriksson, "THE VERY FAST CURVELET TRANSFORM", ECE 734 - VLSI Structures for Digital Signal Processing Final Project Report, 2007 